\title{
MÁQUINAS TÉRMICAS DESDE LA ANTIGÜEDAD AL SIGLO XVII: ANÁLISIS HISTÓRICO DESDE LA FILOSOFÍA DE LA TÉCNICA
}

\author{
LEANDRO GiRI \\ IIF-SADAF/CONICET - UNAJ - UNTREF Argentina
}

\section{Resumen}

En el presente trabajo se propone un análisis histórico, apoyado en el marco propuesto por Miguel Ángel Quintanilla en su filosofía de la técnica, de una genealogía de máquinas térmicas desde la antigüedad hasta el siglo XVII. Se espera así trazar una línea histórica que permita mostrar una continuidad relevante entre dichos artefactos y las máquinas térmicas contemporáneas, cuya historia, afirmamos, a diferencia de lo que suele proponerse en la bibliografía al respecto, no comienza en la máquina de vapor sino mucho antes. La filosofía sistémica de la técnica de Quintanilla permitirá iluminar las estructuras de los sistemas técnicos de los cuales los artefactos a analizar forman parte, y así argumentar que son antecedentes necesarios a las máquinas térmicas contemporáneas. En tal sentido, afirmamos que los análisis históricos sobre las máquinas térmicas contemporáneas y sobre la termodinámica se verían enriquecidos por la inclusión de la línea genealógica de artefactos aquí presentados.

\section{Abstract}

In the present article we present a historical analysis, supported by the framework proposed by Miguel Ángel Quintanilla in his philosophy of technique, of a genealogy of thermal machines from antiquity to 17 th century. We intend to draw a historical line that allow us to show a relevant continuity between such artifacts and contemporaneous thermal machines, whose history, we argue, do not start with the steam machine but much earlier. Quintanilla's systemic philosophy of technique allows to enlighten the structures of the technical systems in which the artifacts to analyze are embedded, in order to argue that they are necessary antecedents to contemporary thermal machines. In that sense, we affirm that historical analysis about contemporary thermal machines and about thermodynamics would be enriched by the inclusion of the genealogic line of artifacts here presented.

Recibido el 10 de febrero de 2020 - Aceptado el 5 de junio de 2020

https://doi.org/10.47101/llull.2020.43.87.02giri

TLITL, Vol. 43 (N.o 87) 2020 - ISSN: 0210-8615, pp. 29-43 
Palabras claves: Máquinas térmicas, Historia medieval de la técnica, Filosofía sistémica de la técnica.

Key words: Thermal machines, Medieval History of Technique, Systemic Philosophy of Technique.

\section{INTRODUCCIÓN}

En el presente trabajo nos proponemos una indagación de carácter histórico y filosófico sobre las máquinas térmicas.

El concepto de máquina térmica no es controversial. Cualquier tratado básico en termodinámica provee dicha noción de manera similar. Tomemos por caso la definición provista por Cenguel y Boles [2009, p. 284]:

Las máquinas térmicas difieren bastante entre sí, pero es posible caracterizarlas a todas (...) [como cumpliendo los siguientes requisitos]

1- Reciben calor de una fuente a temperatura alta (energía solar, horno de petróleo, reactor nuclear, etc.)

2- Convierten parte de este calor en trabajo (por lo general en la forma de una flecha rotatoria).

3- Rechazan el calor de desecho hacia un sumidero de calor de baja temperatura (la atmósfera, los ríos, etc.)

4- Operan en un ciclo.

Las máquinas térmicas y otros dispositivos cíclicos por lo común requieren un fluido hacia y desde el cual se transfiere calor mientras experimenta un ciclo. Al fluido se lo conoce como fluido de trabajo.

Cabe sin embargo aclarar que:

El término máquina térmica se usa con frecuencia en un sentido más amplio que incluye dispositivos que producen trabajo que no operan en un ciclo termodinámico. Las máquinas relacionadas con la combustión interna, como las turbinas de gas y los motores de automóviles, entran en esta categoría. Estos dispositivos operan en un ciclo mecánico pero no en un ciclo termodinámico, porque el fluido de trabajo (los gases de combustión) no experimentan un ciclo completo. En lugar de ser enfriados a la temperatura inicial, los gases de escape se purgan y se reemplazan por una mezcla fresca de aire y combustible al final del ciclo. [Id, énfasis propio].

Resulta conveniente, antes de comenzar una indagación precisa en el tópico de las máquinas térmicas, presentar un marco conceptual que nos permita ubicarlas dentro de un cuadro más general de los sistemas técnicos. De ese modo, podremos identificar con mayor facilidad a qué entidades puede legítimamente llamárseles "máquinas térmicas", lo que resultará de gran utilidad en la indagación histórica que nos proponemos. El marco que seleccionamos será, dada su gran precisión y fertilidad, el de la filosofía sistémica de Miguel Ángel Quintanilla [1998; 2005].

En dicho marco, la unidad de análisis es el "sistema técnico", el cual comprende el todo complejo formado por el artefacto, los materiales, la energía y los usuarios. Para ser más específicos respecto a los componentes del sistema técnico, citaremos in extenso: 
A- Componentes materiales: se trata de las materias primas que se utilizan y se transforman en el sistema técnico (...), la energía que se emplea para las operaciones del sistema (...), y el equipamiento, es decir, los componentes técnicos del propio sistema (...).

B- Componentes intencionales: (...) Los agentes de un sistema técnico son generalmente individuos humanos, caracterizados por sus habilidades, sus conocimientos y valores (...) y que actúan en el sistema bien sea como usuarios, como operadores manuales o como controladores o gerenciadores del sistema (...).

C- La estructura del sistema: Está definida por las relaciones o interacciones que se producen entre los componentes del sistema. Distinguimos dos tipos: relaciones de transformación y relaciones de gestión. Entre las primeras cabe distinguir los procesos físicos que se producen en los componentes materiales del sistema, por una parte, y las acciones de manipulación que llevan a cabo los agentes intencionales. (...) Las relaciones de gestión son también relaciones entre los componentes del sistema, pero en ellas lo que cuenta no son las transformaciones materiales que se producen entre los componentes, sino el flujo de información que permite el control y la gestión global del sistema (...). D- Los objetivos: (...) Para caracterizar un sistema técnico es muy importante definir bien sus objetivos, a ser posible en términos precisos y cuantificables. (...)

E- Los resultados: (...) para caracterizar y valorar cualquier sistema técnico, es importante distinguir entre los objetivos previstos y los resultados realmente obtenidos. [QUiNTANILLA ,1998, pp. 53-55]

Presentados estos conceptos podemos precisar de manera amplia a los objetos técnicos de interés, de modo de considerar la unidad de análisis de la filosofía sistémica de la técnica de Quintanilla. Así, no nos interesará la "máquina" en sentido artefactual (más propio de análisis de tipo instrumental de la técnica [Quintanilla, 2005], sino el sistema técnico del que ésta forma parte.

Los sistemas técnicos de interés poseerán entonces los siguientes componentes interrelacionados:

A. Con respecto a los componentes materiales, como mínimo deben cumplirse los siguientes requisitos: existirá entre las materias primas al menos un fluido de trabajo (hacia y desde el cual se transfiere calor). Entre los componentes energéticos habrá una fuente de calor y un sumidero de calor (fuente fría). Estas fuentes muchas veces no se encuentran dentro del componente técnico del sistema (el artefacto propiamente dicho) pero son parte fundamental del sistema técnico, dado que sin él no funciona el sistema en el sentido deseado.

B. El/los agente/s intencional/es manipula/n el sistema técnico con el fin de lograr los objetivos deseados (esto es trivialmente cierto en todo sistema técnico).

C. En los sistemas técnicos de interés las relaciones de transformación son tales que parte de la energía calórica provista por la fuente caliente se transforma en energía mecánica (trabajo), mientras la restante se disipa hacia el sumidero o fuente fría.

D. El objetivo implícito del sistema técnico de interés es obtener trabajo mecánico. El objetivo explícito estará relacionado con el efecto puntual del cual el trabajo mecánico realizado sobre algún determinado componente técnico del sistema es la causa.

E. El conjunto de resultados debe poseer al menos parte de los objetivos, de modo de que el sistema sea al menos mínimamente eficaz y eficiente. 
Llamaremos entonces a los sistemas técnicos que cumplan los requisitos A-E "sistemas técnicos térmicos".

El ejemplo paradigmático de sistema técnico térmico es la planta termoeléctrica. Para analizar cómo tal ejemplo es compatible con la definición propuesta nos centraremos en los componentes no-triviales de la caracterización: los componentes materiales (a), la estructura del sistema (b) y los objetivos (c).

a. Entre los componentes materiales hay un fluido de trabajo que es agua. Esta es llevada a temperatura ambiente hacia una caldera, donde se le provee calor desde una fuente caliente (un reactor de combustión que puede ser alimentado por combustible fósil (derivado de petróleo o gas natural) o por un combustible nuclear). El agua cambia de estado en la caldera y se transforma en vapor caliente a presión de caldera, el cual se lleva a un componente técnico donde ocurrirá la relación de transformación del calor a trabajo (en general una turbina), y luego el vapor, ya con menor presión y temperatura, es conducido a una fuente fría (generalmente una torre de enfriamiento) para su condensación y reinicio del ciclo.

b. El vapor se expande en la turbina, reduciendo su presión y temperatura. Ocurre la relación de transformación, realizándose un trabajo mecánico (que produce que la turbina rote).

c. El objetivo explícito del sistema es la generación de energía eléctrica. Sin embargo, lo que causa tal generación es el trabajo mecánico de la rotación de la turbina, el objetivo implícito del sistema.

La máquina de vapor clásica forma parte de un sistema técnico térmico como el descrito, ya que funciona como una suerte de planta termoeléctrica en miniatura, donde la única diferencia sutil sería en el objetivo explícito del sistema: en los tiempos en que no existía la tecnología de aprovechamiento de la energía eléctrica, las máquinas de vapor eran utilizadas para lograr efectos directos del trabajo mecánico entregado (para bombear agua dentro de minas, para impulsar barcos, para mover telares, etc.)

La máquina de vapor más antigua de que se tiene registro sería de 1606, habiendo sido registrada por su inventor, el español Jerónimo de Ayanz y Beaumont [GARCía TAPIA, 2010]. La primera máquina con la concepción de diseńo moderna será la de James Watt de 1774, la cual a su vez fue una mejora sobre la de Thomas Newcomen de 1712 .

Al trazar una genealogía que dé cuenta de la evolución de estos artefactos se mencionan por ejemplo a las esferas de Magdeburgo (semiesferas que se unían mediante un sello hermético y eran sometidas a vacío, resultando imposibles de separar, ni siquiera con la fuerza de una tropilla de caballos tirando en direcciones opuestas) de Otto von Guericke (1654), las cuales permitieron iniciar el camino a la concepción contemporánea acerca del comportamiento del aire y del vacío y de las posibilidades de utilización energéticas de los mismos [JACOMY (1992)]. Entre los que propusieron distintas versiones cada vez mejores de la máquina de vapor, se suele mencionar a ingenieros y científicos como Papin, Savery, Huygens, Leibniz y 
otros: todos ellos posteriores a la máquina original del navarro Ayanz y Beaumont, es decir, posteriores al siglo XVI.

No es intención del presente trabajo analizar en profundidad la historia de la máquina de vapor, habiendo sido esta objeto de muy profundas indagaciones históricas [JАСОмY, 1992; Ducassé, 1960; Rosen, 2010], fomentadas por ser dicha máquina la responsable de la Revolución Industrial del siglo XIX. Más bien, aquí el objetivo es utilizar el marco de la filosofía de la técnica de Quintanilla para darle entidad a una serie de invenciones, que van desde la antigüedad hasta el siglo XVII, que constituyen legítimamente sistemas técnicos térmicos, y por ende son antecedentes virtuosos de la máquina de vapor.

Existe una tesis continuista en historia de la ciencia (cuyo principal exponente fue Aleistar Crombie [1953], que asevera, en la línea de Pierre Duhem, que la Edad Media fue fértil en la generación de conocimiento científico, restando importancia relativa a la revolución científica galileana. Por otra parte, existe también una tesis discontinuista defendida por Alexandre Koyré [1978] y sus seguidores, quienes marcan a Galileo como un parteaguas en la historia de la ciencia. Pues bien, aquí sostendremos una tesis "continuista" en la historia de la técnica, en particular en la línea de los sistemas técnicos térmicos, pues afirmamos que la importancia atribuida a la máquina de vapor para la evolución técnica de las máquinas térmicas oscurece un pasado virtuoso de invenciones relevantes en dicha evolución. Por ello, nos proponemos explorar algunos sistemas técnicos emblemáticos anteriores a la creación de la máquina de vapor, demostrando su pertinencia gracias al marco conceptual presentado.

El objetivo de este análisis, en otras palabras, es comprender la dinámica de la cultura técnica que llevó a las máquinas térmicas modernas. Quintanilla afirma que la cultura técnica incorporada a los sistemas técnicos se caracteriza por sus

...Componentes cognitivos, representacionales o simbólicos: conocimientos técnicos y científicos aplicados; componentes prácticos u operacionales: reglas de operación, habilidades técnicas de diseño, producción y uso de artefactos, y componentes valorativos: objetivos incorporados a los sistemas técnicos y valoración de sus resultados (...) [QuintaniLla, 1998, p. 61]

En muchas historias de la técnica, cuando se menciona la historia de las máquinas térmicas se comienza con la máquina de vapor, o a lo sumo con la eolipila de Herón de Alejandría, de la cual nos ocuparemos más adelante [Rosen, 2010], pero más como una curiosidad histórica que como un antecedente virtuoso y necesario. Por ejemplo,

Estos (...) dispositivos, que ahora llamaremos máquinas térmicas, se desarrollaron desde su forma más incipiente, en el siglo XVIII, hasta prácticamente la forma en que las conocemos hoy en día, lo que ocurrió ya hacia mediados del siglo XIX. [GARCía y SCHERER 1997, p.4]

Sin embargo, existen componentes de la cultura técnica previa a la máquina de vapor que es necesario tener en cuenta para una descripción completa de la dinámica histórica, ya que han aportado ciertos elementos clave para la génesis de dicha máquina. Además de determinados componentes cognitivos y prácticos imprescindibles en la constitución de 
materiales, capaces de soportar altas temperaturas y de ciertas capacidades para utilizar la fuerza expansiva del vapor y otros gases calientes para generar trabajo mecánico (a través de distintos dispositivos como palancas, engranajes y ruedas), aparecen también valores asociados al aprovechamiento de las fuerzas de la naturaleza para el beneficio de la humanidad. Estos valores son fundamentales para entender los esfuerzos por ahorrar esfuerzos humanos que llevaron a las máquinas térmicas actuales. Así, afirmamos que de no tener en cuenta estos componentes de la cultura técnica aportados sobre todo por el pensamiento medieval, no es posible comprender correctamente la dinámica de la técnica que llevó a la máquina de vapor, prevaleciendo la imagen de su surgimiento como habiendo sido desde una tabula rasa del conocimiento técnico, cosa que negamos rotundamente. Si bien no compartimos una posición de determinismo tecnológico, sí creemos en la influencia profunda de ciertos antecedentes en la creación de algunos avances científicos y técnicos fundamentales, y entendemos la indagación histórica como la herramienta específica para arrojar luz sobre tales influencias.

En cierto sentido, nuestra tesis no es totalmente original. Posee (al menos) un antecedente fundamental en el gran trabajo de Lynn Townsend White Jr. [1974], quien indagó de manera profunda en la exploración de la energía y los dispositivos mecánicos, especialmente en la Baja Edad Media. El trabajo de White es más abarcativo y amplio que el aquí presentado, ya que analiza una multiplicidad de artefactos que aprovechan distintas fuerzas de la naturaleza, como molinos eólicos o hidráulicos, además de otros que efectivamente entran dentro de los que nos interesan aquí. White muestra (a nuestro entender exitosamente) que la actitud moderna de conquista de la naturaleza para el beneficio humano no expresa una completa novedad sino que ya formaba parte de las ambiciones de los ingenieros medievales que ya habían, con mayor o menor grado de eficiencia, logrado notables resultados en ese sentido. Nuestra intención es menos ambiciosa, pues simplemente queremos resaltar que la historia de los sistemas técnicos térmicos no es una historia que comienza con la máquina de vapor hasta convertirse en una tecnología con el advenimiento de la termodinámica teórica y su aplicación al perfeccionamiento de los artefactos existentes. Pretendemos, como ya afirmamos, simplemente esbozar una línea "continuista" que le dé a los ingenieros medievales el lugar que se merecen en la historia de la técnica, y a su vez proveer una casuística que permitiría enriquecer los abordajes históricos a las máquinas térmicas contemporáneas y a la termodinámica como disciplina científica.

\section{Sistemas téCNicos térmicos desde la ANTIGÜEDAd}

Existe consenso en atribuirle el honor de ser la primera máquina térmica registrada a la eolipila de Herón de Alejandría en el siglo I dC [White, 1974; García y Scherer, 1997; DrachmanN, 1948] y, en este sentido, es la única máquina antigua que suele ser mencionada en las historias de la máquina de vapor como antecedente relevante. La eolipila (ver figura 1) consiste de una esfera metálica hueca rellena de agua, que puede girar alrededor de un eje horizontal, hallándose provisto de dos tubos curvos que parten de los extremos de otro diámetro, perpendicular al primero. El hecho de que los tubos estén curvados de manera 
conveniente provoca un movimiento de rotación causado por el vapor de agua que se escapa por los mismos al ser calentada la esfera [Delaunay, 1864] ${ }^{1}$. El objeto de semejante artefacto era lúdico, pues sería entretenido en aquella época observar algo capaz de girar por sí mismo, sin una fuerza externa directamente impresa. Presumiblemente, la eolipila se ha ganado su lugar en la historia por la influencia del Pneumatica de Herón en la pre-teorización de la termodinámica durante el renacimiento tardío [TRUITT, 2015], ya que fue recuperado y traducido al latín en 1575 por Comandino [SARTON, 1927], pero parece haber tenido escasa influencia sobre la cultura técnica de la Edad media [BOAs, 1949; Rosen, 2010].

Cabe preguntarse si la eolipila es stricto sensu una máquina térmica. La respuesta es que efectivamente lo es, dado que recibe calor de una fuente caliente (una llama), rechaza el calor hacia una fuente fría (el aire circundante), posee un fluido de trabajo (el agua interna a la esfera) y genera un trabajo (para llevar la esfera desde el reposo a su velocidad de rotación, y luego en la variación de la velocidad y contra el rozamiento de la esfera contra el eje). Por supuesto, si es una máquina térmica también forma parte de un sistema técnico térmico, pero en este sentido, habría que integrar como parte del mismo al recipiente donde se coloca el combustible para generar la llama (fuente caliente), al aire de la atmósfera circundante donde el vapor de agua de la eolipila descarga su calor y produce la expansión, a los agentes intencionales y sus objetivos (el explícito que es lúdico y el implícito que es la generación del trabajo de rotación de la esfera) y resultados.

Ahora bien, existen otros sistemas técnicos térmicos que son relevantes en una genealogía técnica de la máquina de vapor, que no son usualmente tenidos en cuenta a la hora de relevar los antecedentes de esta en los análisis históricos, y procederemos a relevarlos. El recorte histórico abordará, más allá de la mención de la eolipila y algunos avances modernos, a la Edad Media, ya que supuestamente, si bien "la fuerza expansiva del vapor caliente ya era conocida en la época helenística, (...) durante más de mil ańos se hicieron escasos intentos por aprovecharla" [WHite, 1974, p.89]. Veremos algunos de esos intentos.

Para continuar nuestra genealogía, abordaremos al artefacto denominado sufflator por Alberto Magno en el siglo XIII (aunque es mucho más antiguo, ver nota 1). En De meteoris describe a esta vasija de barro antropomórfica a la cual se le pone agua y se tapan sus orificios con tapones que saltan al calentarse mediante una llama, desparramando agua caliente y vapor [White, 1974]. El sufflator (figura 2) tiene las características para ser denominado "máquina térmica", pero el sistema técnico conformado por el sufflator no es un sistema técnico térmico, ya que si bien se cumplen algunos de los requisitos (una fuente caliente: la llama; una fuente fría: el aire circundante; un fluido que se expande y realiza un trabajo: sobre los tapones del sufflator que son eyectados por la presión del vapor), el objetivo del sistema no tiene que ver con el aprovechamiento de dicho trabajo mecánico, sino con el avivamiento de llamas en ambientes húmedos, al estilo de un fuelle.

Sin embargo, existen versiones del sufflator que sí son utilizados con el objetivo de aprovechar el trabajo mecánico (figura 3), como el fuelle que hace girar un asador (sugerido por ejemplo por Leonardo da Vinci en su Códice Leicester), o algunos sistemas técnicos sugeridos por John Wilkins en su Mathematicall Magick [1691]. 


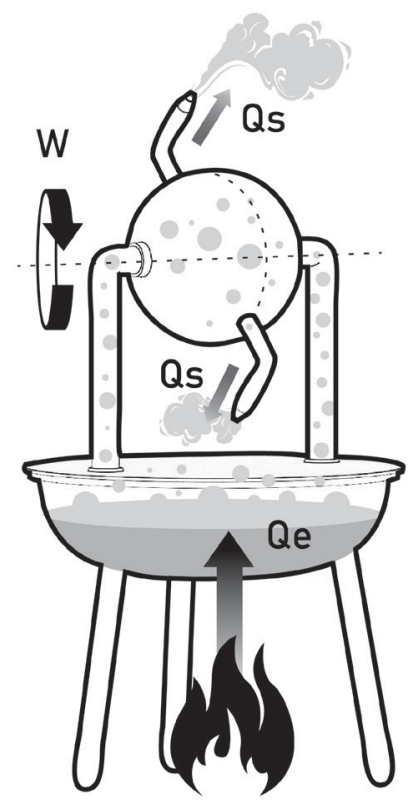

Figura 1. La eolipila de Herón. Qe es el calor de entrada, Qs el calor de salida y W es el trabajo de rotación de la esfera (diagrama de elaboración propia)

Es curioso que Wilkins llama al componente técnico de los sistemas que analiza "eolipilas" aunque los describe como

... un recipiente cóncavo consistente en un material tal que pueda resistir el fuego, poseyendo un pequeño agujero, a través del cual son llenados con agua, y por el cual sale (cuando el recipiente es calentado) el aire hacia adelante con fuerte y duradera violencia. Son frecuentemente utilizados para el excitamiento y contracción del calor en el derretimiento de vidrios o metales [WILKINs, 1691, p.149].

Parece claro que no estamos hablando de eolipilas como las que pensó Herón sino de sufflators. Sin embargo, lo interesante en este caso es que Wilkins plantea no su uso "tradicional" como fuelle sino como parte de un sistema técnico donde el aire expulsado por el sufflator (o la chimenea de un hogar) acciona un componente mecánico (como una vela solidaria con una rueda) lo cual permite aprovechar el trabajo con objetivos variados como sonar campanillas u otros artefactos musicales ("no puede haber una estratagema más placentera para tener una música continua y barata" [WiLKINs, 1691, pp. 151-52]), como así también para bobinar hilo o mecer cunas. En todos los casos, estamos hablando de sistemas técnicos térmicos con el sufflator como componente técnico central. Puede verse entonces la ventaja del enfoque sistémico de Quintanilla para nuestro análisis, ya que el mismo artefacto 
formó, a lo largo de la historia, parte de sistemas técnicos muy distintos, lo cual permite notar que estos sistemas pertenecen a una misma familia a pesar de tales diferencias.

Giovanni Branca en su Le Machina de 1629 [KelLer, 1978] y Atanasio Kircher en su Magnes, sive de arte magnetica de 1641 [MerriLl, 2003] presentan también sistemas técnicos térmicos con turbinas y pistones cuyo componente técnico son sufflators. No profundizaremos en estos pues nuestro interés radica en las invenciones anteriores a las máquinas de vapor primigenias. Sin embargo, queda clara la importancia del análisis de este artefacto dentro de una genealogía de los sistemas técnicos térmicos.

Un último tipo de sistema sobre el cual queremos indagar en este trabajo, será el relacionado con la cuestión bélica, en cohetes y armas de fuego. En ambos casos, se produce una reacción de combustión violenta (por la quema de un combustible), que libera energía calórica oficiando de fuente caliente. Los gases de combustión en expansión ofician de fluido de trabajo y, al escapar hacia la fuente fría (el aire circundante), generan trabajo mecánico que impulsa a un proyectil (figura 4). El objetivo implícito es generar dicho trabajo mecánico, mientras que el explícito es o bien lúdico (como en el caso de los fuegos artificiales) o bien bélico.

La indagación sobre estos artefactos no será profunda, pues existen múltiples y muy profundos análisis históricos de las armas de fuego [PARTington, 1999; VAN Riper, 2004; Pauly, 2008; Chase, 2003]. Simplemente haremos mención a algunas variaciones de estos con el fin de encontrar características técnicas que permitan incluirlos dentro de la genealogía de los sistemas técnicos térmicos.

La clave de estos dispositivos es la invención y el perfeccionamiento paulatino de la pólvora, una mezcla de carbón, azufre y salitre (nitrato de potasio) surgida en China entre el 500 y el 900 d.C. La receta no era la misma para cohetes y armas, ya que los primeros llevaban una proporción mayor de carbón para que la combustión fuese más lenta y permitiera al cohete ser impulsado durante mayor tiempo. Además, la pólvora en los tubos lanzacohetes eran dispuestas en "tortas" más que en su forma granulada [VAN RIPER, 2004], mientras que en las armas la disposición granular provocaba una explosión violenta para impulsar al proyectil hacia su objetivo. Así, mientras en las armas una explosión en el tubo provoca una expansión de los gases que genera un trabajo que impulsa un proyectil (moviéndose, en teoría, solamente el proyectil), en los cohetes, una combustión más lenta genera una expansión de los gases que a su vez provoca el trabajo de impulsión del propio cohete.

Manuscritos chinos revelan uso de pirotecnia hacia el 600 [CARRINGTON Goodrigde Y Chia-Sheng, 1946; Ling, 1947], y de algo que han dado en llamar "lanzas de fuego" o "flechas de fuego" hacia el 1200 en aplicaciones bélicas, que mientras Van Riper [2004] considera cohetes primitivos, para White [1974] se trataría tan solo de candelas romanas. En la segunda mitad del siglo XIII Roger Bacon menciona cohetes en De secretis operibus, mientras Alberto Magno menciona fuego volador en De Mirabilibus Mundi ("pólvora empacada en un tubo largo y fino"[Van Riper, 2004, p. 9]. El Liber Ignium de Marco el griego posee ya una descripción detallada de cómo fabricar sustancias inflamables para la 


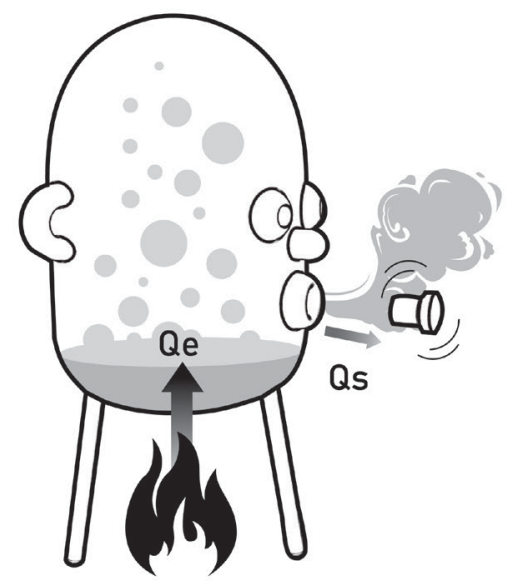

Figura 2. El sufflator, artefacto utilizado para avivar llamas por la eyección de vapor caliente (diagrama de elaboración propia)

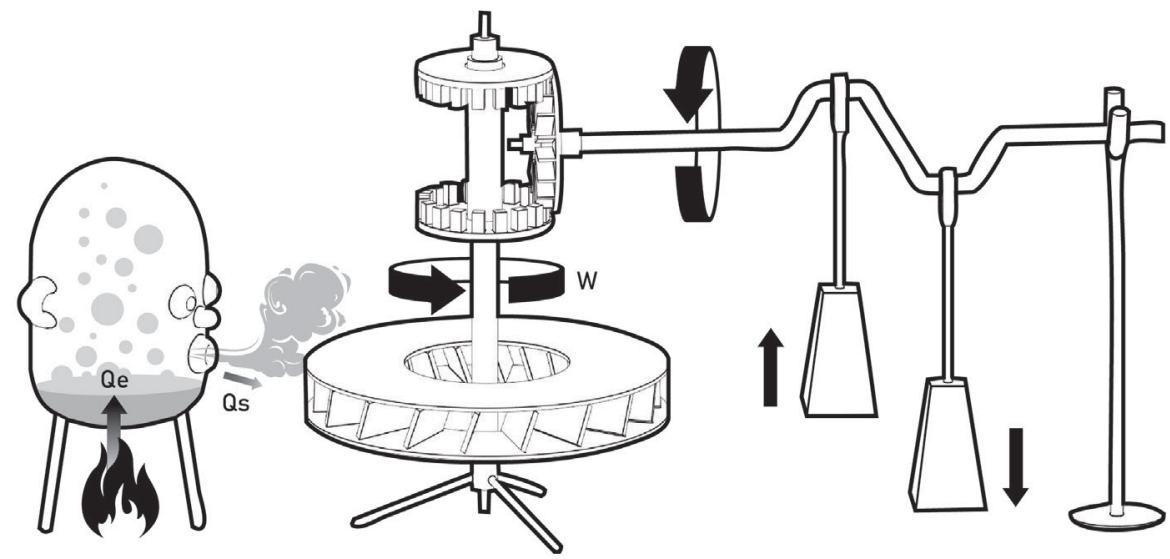

Figura 3. Sistema térmico con un sufflator como componente técnico central (diagrama de elaboración propia)

guerra (incluyendo combustible de cohete [PARTIngton, 1999]), mientras que Al-Hassan al-Rammah en su Tratado sobre equitación y Estratagemas de Guerra

...ofrece sus propias fórmulas junto a descripciones más detalladas de cohetes y armas impulsadas por cohetes. Una de dichas armas es un "huevo que se mueve a sí mismo y quema" propulsado por cohetes, que al-Rammah afirma que fue utilizado por fuerzas árabes contra los franceses en la Batalla de Damiah en 1248. [VAn Riper, 2004, pp. 9-10]. 
Sin embargo, afirma White que

La confusa historia de la aparición de los explosivos y las armas de fuego ha de entenderse como un conjunto de experimentos regionales paralelos, basados fundamentalmente en las diversas formas del fuego griego, y con un intercambio ocasional de técnicas más perfectas a medida que fueron evolucionando los métodos químicos.

A pesar de la sugerencia de Al-Hasan al-Rammāh, la propulsión a chorro para todo lo que no fuesen flechas de fuego no se desarrolló hasta el siglo XV, época en que técnicos italianos empezaron a considerar las posibilidades de aquélla. [WHITE,1974, pp. 97-98]

Giovanni da Fontana es uno de los tantos hábiles italianos que se aficionaron a los cohetes. da Fontana los utilizó para realizar mediciones de alturas y profundidades, y también

... determinó por una serie de experimentos la cantidad de pólvora necesaria para elevar cohetes a diferentes alturas. Sin embargo no sólo muestra cómo disparar un cohete hacia el aire sino también

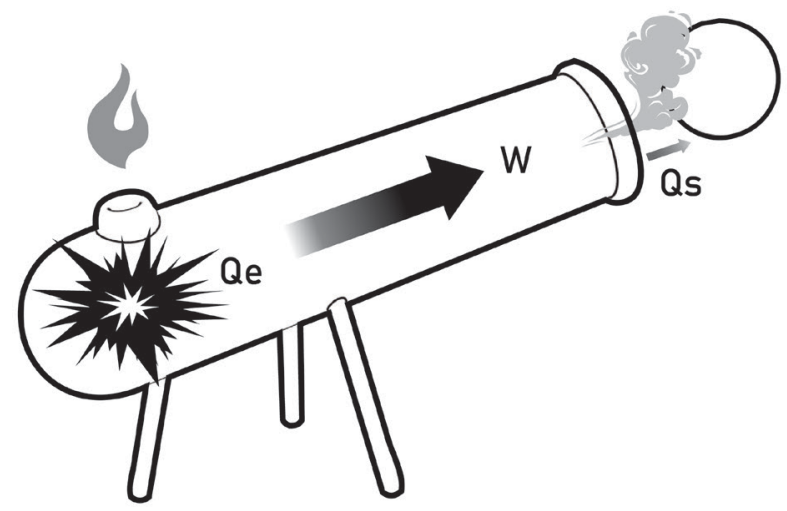

Figura 4. Cañón medieval visto como sistema técnico térmico (diagrama de elaboración propia)

cómo darle la apariencia de un diablo o dragón volador (...). O, en conexión con una clase de torpedo o vela mágica que se quema bajo el agua y alternativamente emerge y se hunde mientras las pólvoras más débiles o más fuertes se quemaban, nos dice que una vez deliberadamente engańó a un monje (...). Construyó una figura diabólica llena de tales químicos que se movía bajo el agua y emitía rayos de fuego que asustaron enormemente al monje [Thorndike, 1934, p. 174].

Otros inventos de Fontana fueron un ariete naval y un tanque militar impulsados por cohetes. Similares inventos pergeñó en la misma época Francesco di Giorgio.

Sobre las armas de fuego, afirma White que son un invento occidental, "derivado de la técnica bizantina de lanzamiento de fuego griego desde tubos de cobre" [White, 1974, p. 98]. El avance técnico fundamental es la calibración de los proyectiles, documentado en Inglaterra hacia 1244. El ya mencionado Liber Ignium de Marco el griego también posee 
recetas de pólvora balística para cañones. Múltiples referencias a cañones de alto calibre y armas manuales aparecen hacia mediados del siglo XIV, tanto en Occidente como en China y el mundo Islámico.

La importancia para nosotros de esta tecnología puede deducirse de la siguiente cita de White:

El cańón no sólo es importante en sí mismo como artefacto mecánico aplicado a la guerra: es una máquina de combustión interna de un cilindro, y todos nuestros más modernos motores de ese tipo descienden de aquel. En el primer intento de sustituir la bala de cańón por un pistón, o sea el de Leonardo da Vinci, se utilizó pólvora como combustible, al igual que en el invento patentado por Samuel Moreland en 1661, en la máquina experimental de pistón diseńada por Huygens en 1673 y en una bomba parisiense de aire de 1674 [White, 1974, p. 100].

Como vemos, la cita es elocuente para justificar por qué estos sistemas técnicos térmicos merecen un lugar en la genealogía que nos interesa, como antecedentes relevantes a las máquinas de vapor modernas.

Valga la aclaración, la lista de artefactos indagados en el presente breve artículo no pretende ser exhaustiva, pudiendo haber muchos más de considerable relevancia. La pretensión aquí ha sido más austera: simplemente se advierte que un programa de investigación con un recorrido histórico más amplio puede arrojar luz sobre la cultura técnica medieval y cómo esta dio por origen a las máquinas de vapor modernas (y su consiguiente influencia en una historia de la termodinámica).

\section{Conclusiones}

En el presente trabajo, hemos presentado, utilizando el marco conceptual de la filosofía sistémica de la técnica de Miguel Ángel Quintanilla [1998; 2005] el concepto de "sistema técnico térmico". Dicha conceptualización permite mostrar una genealogía de artefactos que son antecedentes necesarios de las máquinas térmicas modernas, al conectarse con la máquina de vapor, raíz artefactual de toda la tecnología térmica contemporánea.

Hemos presentado, a través del análisis de fuentes historiográficas primarias y secundarias, un conjunto de sistemas técnicos térmicos, a saber: la eolipila, el sufflator (y los sistemas que lo contienen como componente técnico) y los cañones y armas de fuego, y hemos argumentado que todos ellos son antecedentes necesarios de la máquina de vapor, ya que se trata de sistemas que convierten energía térmica en energía mecánica con algún fin, y por ello merecen su posición dentro de una genealogía de este tipo de artefactos. Así pues, deberían formar parte de una historia moderna de la termodinámica y su tecnología asociada. Como advertimos previamente, no se trata de una lista exhaustiva, pero al menos alcanza para dar la pauta de que no se ha prestado suficiente atención en la investigación histórica a algunos artefactos relevantes en una genealogía de las máquinas térmicas.

En el análisis filosófico sobre el conocimiento tecnológico propuesto por Meijers y de Vries [2013] basado en estudios históricos de Staudenmaier, se sostiene que el mismo, además 
de contener conocimiento científico que le sustenta, contiene "datos empíricos específicos para la tecnología (datos que no son solamente instrumentales para el desarrollo de teorías científicas), teorías tecnológicas y know-how tecnológico (o habilidades)" [MeIJERS y DE VRIES, 2013, p.71]. Si bien para los artefactos que nos han ocupado no encontraremos conocimiento científico (al menos en su forma moderna) sustentando la creación de los mismos (la termodinámica es una creación muy posterior), sí encontraremos al menos una proto-teoría tecnológica la cual, argumentamos aquí, es plausible que haya contribuido a la creación de las máquinas térmicas modernas. La teoría sistémica de Quintanilla ha cumplido su papel al permitirnos, a través del análisis de los artefactos, abstraer la estructura de esta proto-teoría. La misma (en términos de la Física contemporánea) establece que es posible aprovechar la energía que es transportada por un fluido de trabajo desde una fuente caliente a una fuente fría para generar un trabajo mecánico. Claro está que le llamamos proto-teoría y no teoría porque los términos que abstragimos no habían sido aún acuñados en los tiempos de nuestro recorte, pero la idea del aprovechamiento de las fuerzas térmicas presente en forma fenomenológica o tácita plausiblemente ha servido de germen para la posterior creación de las teorías modernas. Es en este sentido que sostenemos que estos artefactos merecen formar parte de una historia de las máquinas de vapor y de la termodinámica.

De este modo, hemos echado luz sobre la dinámica de la cultura técnica asociada a las máquinas térmicas, mostrando que un recorte histórico que parta desde la antigüedad resulta relevante, pues una serie de conocimientos y valores se gestaron allí y han sido necesarios para la concepción moderna de las mismas.

\section{Notas}

1. La eolipila debida a Herón es aquella en que la salida de vapor por sus toberas produce trabajo rotatorio en el recipiente esférico. Sin embargo, en varios textos antiguos y medievales aparece la mención a la eolipila simplemente como un recipiente al cual se llena de agua que al calentarse escapa de forma violenta hacia el exterior por uno o varios orificios, sin realizar un trabajo mecánico relevante. A este artefacto se le ha llamado sufflator y lo trataremos más adelante en detalle. Cabría dudar de que haya sido Herón el creador del sufflator. La datación de la vida de Herón es consensuada en el siglo I d.C. (debido al análisis de Neugebauer del Dioptra de Herón, donde parece mencionar un eclipse lunar ocurrido en el año 63 DC [Boas (1949)], mientras existe, por ejemplo, una mención a este artefacto en De Architectura de Vitrubio, escrito entre el 23 y el 27 a.C. "Que esto sea así [la naturaleza del viento] se puede ver en las eolipilas de bronce, y de este artificial invento, inferir la verdad de las cosas ocultas de la naturaleza. Las eolipilas se hacen de bronce, huecas, y tienen un agujero muy angosto por donde se llenan de agua: expuestas al fuego, antes de calentarse, no despiden, ayre alguno, pero luego que toman calor arrojan vehementísimo viento" [Vitrubio, 1787, p. 20].

En esta mención de Vitrubio, no hay mención a que la eolipila gire o siquiera sea esférica, por lo que deducimos que se trata de un sufflator.

\section{REFERENCIAS BIBLIOGRÁFICAS}

Boas, M. (1949) "Hero's Pneumatica: a Study of its Transmission and Influence”. Isis, 40(1), 38-48. Carrington Goodrigde, L., Chia-Sheng, F. (1946) "The Early Development of Firearms in China”. Isis, 36(2), 114-123. 
Cengel, Y., Boles, M. (2009) Termodinámica. Sexta edición, México D.F., Mc-Graw-Hill. Traducción de la $\sigma^{a}$ edición en inglés, 2008.

Chase, K. (2003) Firearms: a Global History to 1700. Cambridge, Cambridge University Press.

Crombie, A. (1953) Robert Grosseteste and the Origins of Experimental Science: 1100-1700. Oxford, Clarendon Press.

Delaunay, M. (1864) Curso elemental de mecánica teórica y aplicada. Madrid, Universidad Complutense.

Drachmann, A. (1948) Ktesibios, Philon and Heron: a Study on Ancient Pneumatics. Copenhaguen, Munksgaard.

Ducassé, P. (1960) Historia de las técnicas. Buenos Aires, Eudeba.

García, L., Scherer, C. (1997) De la máquina de vapor al cero absoluto (calor y entropía). Segunda edición, México D.F., Fondo de Cultura Económica.

García Tapia, N. (2010) Jerónimo de Ayanz y Beaumont, Un Inventor Navarro. Navarra, Universidad de Navarra.

Jасомy, B. (1992) Historia de las técnicas. Buenos Aires, Losada. Traducción de la $1^{a}$ edición en francés, 2008.

Keller, A. (1978) "Renaissance Theaters of Machines". Technology and Culture, 19(3), 495-508.

KoYré, A. (1978) "Los orígenes de la ciencia moderna: una interpretación nueva". En: A. Koyré (ed.) Estudios de historia del pensamiento científico. Segunda edición, México D.F., Siglo Veintiuno, 5175. Traducción de la $1^{\text {a }}$ edición en francés, 1973.

LinG, W. (1947) “On the Invention and Use of Gunpowder and Firearms in China”. Isis, 37(3), 160178.

Mejuers, A., de Vries, M. (2013) “Technological Knowledge”. En: J. Friis, S. Pedersen, V. Hendricks, (eds.) A Companion to Philosophy of Technology. Malden, Wiley-Blackwell. p. 70-74.

Merrill, B. (2003) Atanasius Kircher (1602-1680) - Jesuit Scholar. Mansfield Centre, Martino Publishing.

Partington, J. (1999) A History of Greek Fire and Gunpowder. Baltimore, Johns Hopkins University Press.

Pauly, R. (2008) Firearms: the Life Story of a Technology. Baltimore, Johns Hopkins University Press.

Quintanilla, M.A. (1998) "Técnica y cultura". Teorema, XVII(3), 49-69.

Quintanilla, M.A. (2005) Tecnología: un enfoque filosófico y otros ensayos de filosofía de la tecnología. México D.F., Fondo de Cultura Económica.

Rosen, W. (2010) The Most Powerful Idea in the World: A Story of Steam, Industry and Invention. New York, Random House.

SARTON, G. (1927) Introduction to the History of Science, Vol I: From Homer to Omar Khayyam. Baltimore, Carnegie Institution of Washington.

Thorndike, L. (1934) A History of Magic and Experimental Science, Vol IV. New York, Columbia University Press.

Truitt, E. (2015) Medieval Robots: Mechanism, Magic, Nature and Art. Philadelphia, University of Pennsylvania Press.

VAn Riper, A. (2004) Rockets and Missiles: the Life Story of a Technology. Westport, Greenwood Press.

Vitrubio (1787) De Architectura. Madrid, Imprenta Real. Traducción del latín del original, circa 15 a.C. 
White, L. (1974) Medieval Technology and Social Change. London, Oxford University Press.

WiLkins, J. (1691) Mathematical Magick or the Wonders that may be Performed by Mechanical Geometry. Cuarta Edición, London, Baldwin. 